\title{
GENETIC DIVERSITY AND POPULATION STRUCTURE AMONG 98 MAIZE INBRED LINES INVESTIGATED WITH SSR MARKERS
}

\author{
ZHANG, C. Y. ${ }^{1,2 \Psi}-$ WU, S. W. ${ }^{3 \Psi}-$ HUSSAIN, K. ${ }^{4 *}$ FAN, M. X. ${ }^{1}-$ LIU, C. ${ }^{1}-$ MA, W. J. ${ }^{1}-$

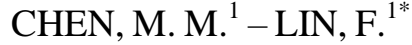 \\ ${ }^{I}$ Biotechnology and Bioscience College, Shenyang Agricultural University \\ No. 120 Dongling Road, Shenyang, Liaoning 110866, China \\ ${ }^{2}$ Agronomy College, Shenyang Agricultural University \\ No. 120 Dongling Road, Shenyang, Liaoning 110866, China \\ ${ }^{3}$ College of Science Institute, Shenyang Agricultural University \\ No. 120 Dongling Road, Shenyang, Liaoning 110866, China \\ ${ }^{4}$ Department of Botany, University of Gujrat \\ HH Campus, Gujrat, Pakistan \\ ${ }^{\Psi}$ Authors have equal contribution to this paper. \\ *Corresponding authors \\ e-mail:fenglinsn@126.com; khalid.hussain@uog.edu.pk \\ (Received $17^{\text {th }}$ Apr 2017; accepted $26^{\text {th }}$ Jun 2017)
}

\begin{abstract}
The conversion of exotic germplasm into domestic maize breeding materials is essential to solve the narrow genetic base for maize improvement in China. The present study applied SSR markers and three complementary cluster methods (STRUCTURE, UPGMA and PCoA) to 98 foreign hybridselected lines for effective hybrid breeding. A total of 450 alleles, with an average of 3.98 alleles per locus, were detected. Among 10 chromosomes, there were 13-19 loci and 33-59 alleles with averaged alleles 2.54 3.43 per chromosome. The polymorphism information content (PIC) among 10 chromosomes ranged from 0.4504 0.5582 and 69.4\% PIC variation were explained by allele number/Locus. The highest PIC was observed in chromosome 7 (0.5582) and the lowest in chromosome 8 (0.4965). The STRUCTURE clustering analysis grouped the test lines into four subpopulations (i.e. REID, Lancaster, P and Domestic) in accordance with UPGMA and PCoA clustering. The higher genetic diversity were detected among the inbred lines in $\mathrm{P}$ and Domestic subpopulations. The allele frequencies, gene diversity and population structure obtained in the present study lead us to conclude that the 98 inbred lines derived from foreign hybrid-selected lines contain extensive genetic variation and are a valuable resource for Chinese maize breeding. The result obtained in the present study will assist in effective utilization of the lines in Chinese hybrid maize breeding programs.
\end{abstract}

Keywords: maize germplasm, hybrid-selected line, genetic variation, clustering analysis, maize breeding

\section{Introduction}

Knowledge of genetic diversity and population structure among inbred lines and breeding materials is of great importance for maize hybrid breeding. With the popularization and application of maize hybrids over the past years, the recurrent use of a few elite germplasm lines as parental stock has led to a decrease in genetic diversity among maize breeding materials in China. The introduction of exotic germplasm, using its abundant genetic variation and good agronomic traits, is therefore essential to solve the narrow genetic base for maize improvement in China (Wen et al., 2012; Yong et al., 
2013). However, it is necessary to make a comprehensive evaluation on the genetic diversity and population structure of exotic germplasm (Tarter et al., 2004; Šarčević et al., 2008; Živanović et al., 2012).

Molecular markers can be employed to investigate levels of genetic diversity and population structure among maize inbred lines and breeding materials. SSRs, due to its abundant, highly polymorphic, genome specific, codominant in nature, have found application in analyses of genetic diversity, population structure, gene mapping, and assisted selection for maize improvement (Phumichai et al., 2012; Wende et al., 2013; Semagn et al., 2014; Yang et al., 2013; Abakemal et al., 2015).

In the present study, 98 foreign hybrid-selected lines were analyzed using 145 SSR loci distributed over the whole maize genome. Our objectives were to estimate the levels of genetic diversity and population structure. The results will be useful to breeders in selecting the best parental combinations for maize breeding program in China.

\section{Research Design and Methods}

\section{Plant Materials}

The germplasm contained 5 tester lines (Huangzao 4, Dan 340, B73, Qi 319 and Mo17) and 98 maize inbred lines derived from foreign maize hybrid-selected lines (obtained by Liaoning Leiao seed company, China) was used in this study. The pedigree and/or origin information can be found in Appendix 1.

\section{SSR Markers and Genotyping}

Genomic DNA was extracted from approximately $200 \mathrm{mg}$ fresh leaf tissue using the cetyltrimethylammonium bromide (CTAB) method (Saghai-Maroof et al., 1984). A total of 500 SSR primers, which were distributed evenly over the 10 maize chromosomes, were selected and synthesized according to the information available in the MaizeGDB database (http://archive.maizegdb.org/).

PCR amplifications were carried out in $10 \mathrm{~mL}$ reaction volumes containing $1 \mu \mathrm{L}$ template DNA, $2 \mu \mathrm{L}$ each of $2.5 \mathrm{mM}$ primer, $5 \mu \mathrm{L} 2 \times$ Taq Master Mix, $0.1 \mu \mathrm{L}$ of 5 units $\mu \mathrm{L}^{-1}$ Taq DNA polymerase, $0.4 \mu \mathrm{L} 10 \mathrm{mM}$ dNTPs and, $\mathrm{dH} 2 \mathrm{O} 2 \mu \mathrm{L}$. PCR protocols consisted of 32 cycles of $94^{\circ} \mathrm{C}$ for $45 \mathrm{~s}$, an annealing temperature at either 45, 50, 55 or $60^{\circ} \mathrm{C}$ depending on the individual SSR primers for $45 \mathrm{~s}$, and $72^{\circ} \mathrm{C}$ for $60 \mathrm{~s}$, and a final extension step of $72^{\circ} \mathrm{C}$ for $10 \mathrm{~min}$. PCR products were analyzed by $8 \%$ polyacrylamide gel electrophoresis (PAGE) and visualized by silver staining.

\section{Genetic Diversity Analysis}

For each SSR locus, polymorphic bands were scored as 1 or 0 for presence or absence of the bands at the same mobility, respectively. Gene diversity (PIC) was calculated for each marker according to the formula: $\mathrm{PIC}=1-\sum \mathrm{fi} 2$, where fi is the allele frequency for the i-th locus summed across all alleles for that locus. The program PowerMarker v3.25 and Excel was used to calculate allele number, allele frequency, and gene diversity of each locus (Liu et al., 2005). 


\section{Population Structure Analysis}

The STRUCTURE v2.3.3 were employed to assess the population structure of the 98 maize inbred lines using the Bayesian model-based approach (Pritchard et al., 2000). The number of subgroups $(\mathrm{K})$, with each $\mathrm{K}$ repeated five times, was ranged from 1 to 12 , with burn-in of 100,000 and run length of 100,000 . We used the ad hoc criterion $\Delta K$ related to the second order rate of change in the log probability of data $(\operatorname{Ln} P(D))$ to determine the most probable K value (Evanno et al., 2005).

To examine genetic relationships among the 98 maize inbred lines, the data matrices of the genetic similarity were used to create the dendrogram using UPGMA clustering with the computer software NTSYS-pc v2.2 (Rohlf 2009). Principal coordinate analysis (PCoA) was also employed to reveal relationships among the 98 inbred lines using the software JMPversion7.0 (SAS Institute Inc., Cary, NC, USA).

\section{Data Analysis and Results}

A total of 500 SSRs, randomly distributed across the maize genome, were used to evaluate the genetic diversity of the 98 maize inbred lines. Finally, 145 SSRs with clear, stable and specific bands were selected to scored on the 98 lines, with an average of 3.98 alleles per locus (range of 2-7). The PIC for all loci ranged from 0.2130 (umc1271) to 0.8316 (bnlg1666) with an average value of 0.5067 (Appendix 2). The higher PIC values indicated the high variability of SSRs, and also a large genetic difference among the 98 maize inbred lines.

The SSRs among 10 maize chromosomes ranged from 11 to 19 with allele number from 33-59 (Table 1). The highest allele number was detected on chromosome 1 (59 alleles), followed chromosome 6 (51 alleles), and the lowest on chromosome 8 (33 alleles). For all chromosomes, there were 13-19 loci and 33-59 alleles with averaged alleles 2.54 3.43 per chromosome. The PIC among 10 chromosomes ranged from $0.4504 \sim 0.5582$. The highest PIC was observed in chromosome $7(0.5582)$ and the lowest in chromosome 8 (0.4965).

Table 1. Genetic diversity at genome level of 98 maize inbred lines revealed by 145 SSR markers

\begin{tabular}{c|c|c|c|c}
\hline Chrom & No. of Loci & No. of Alleles & $\begin{array}{c}\text { Mean allele } \\
\text { number } \\
\text { (range) }\end{array}$ & PIC \\
\hline 1 & 19 & 59 & 3.11 & 0.4897 \\
2 & 13 & 43 & 3.31 & 0.5336 \\
3 & 14 & 47 & 3.36 & 0.5532 \\
4 & 17 & 51 & 3.00 & 0.5199 \\
6 & 16 & 49 & 3.06 & 0.5036 \\
7 & 16 & 51 & 3.19 & 0.4958 \\
8 & 14 & 48 & 3.43 & 0.5582 \\
9 & 13 & 33 & 2.54 & 0.4504 \\
10 & 12 & 35 & 2.92 & 0.4507 \\
& 11 & 34 & 3.09 & 0.5075 \\
\hline
\end{tabular}


There usually was a positive linear relationship between the polymorphism information content (PIC) and number of alleles within a given range. Simple correlation analysis indicated that the PIC was significantly and positively correlated with the number of alleles $(\mathrm{r}=0.6566, p<0.001)$ (Fig. 1). The allele number/Locus ( $\mathrm{x}$ ) could explain $69.4 \%$ PIC variation (y) estimated by a curvilinear regression equation (y $\left.=0.3286 \ln (\mathrm{x})+0.1551(1<\mathrm{x}<8), \mathrm{R}^{2}=0.6937\right)$.

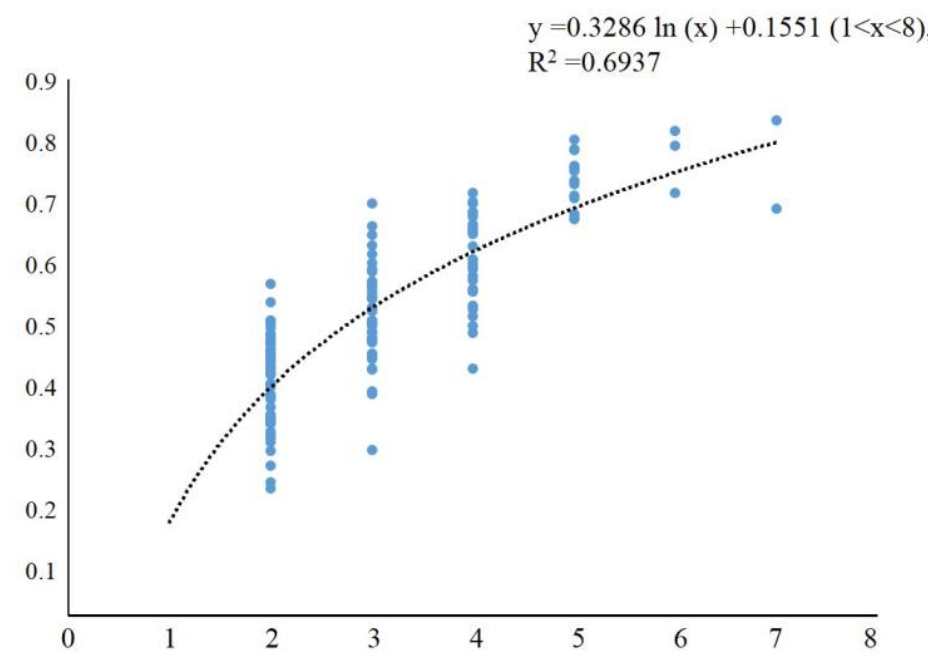

Figure 1. Plot of PIC vs. number of alleles per SSR locus Population Structure Analysis \{tc "5.2.3 Analysis of population structure of mazie core collection" $\backslash$ 000003\}

STRUCTURE V2.3.3 software was employed to assess the population structure of 98 inbred lines based on 145 whole-genome unlinked SSR markers. $\operatorname{Ln} P(D)$ progressively increased as $\mathrm{K}(2-10)$ increased and no obvious inflexion point was observed, which $\operatorname{Ln} P(D)$, in this case, may not be suitable to estimate the true $\mathrm{K}$ value (Fig. 2). The peak of $\Delta \mathrm{K}$ was observed at $\mathrm{K}=4$, suggested that the 98 inbred lines were fell into four sub-populations (Fig. 2).

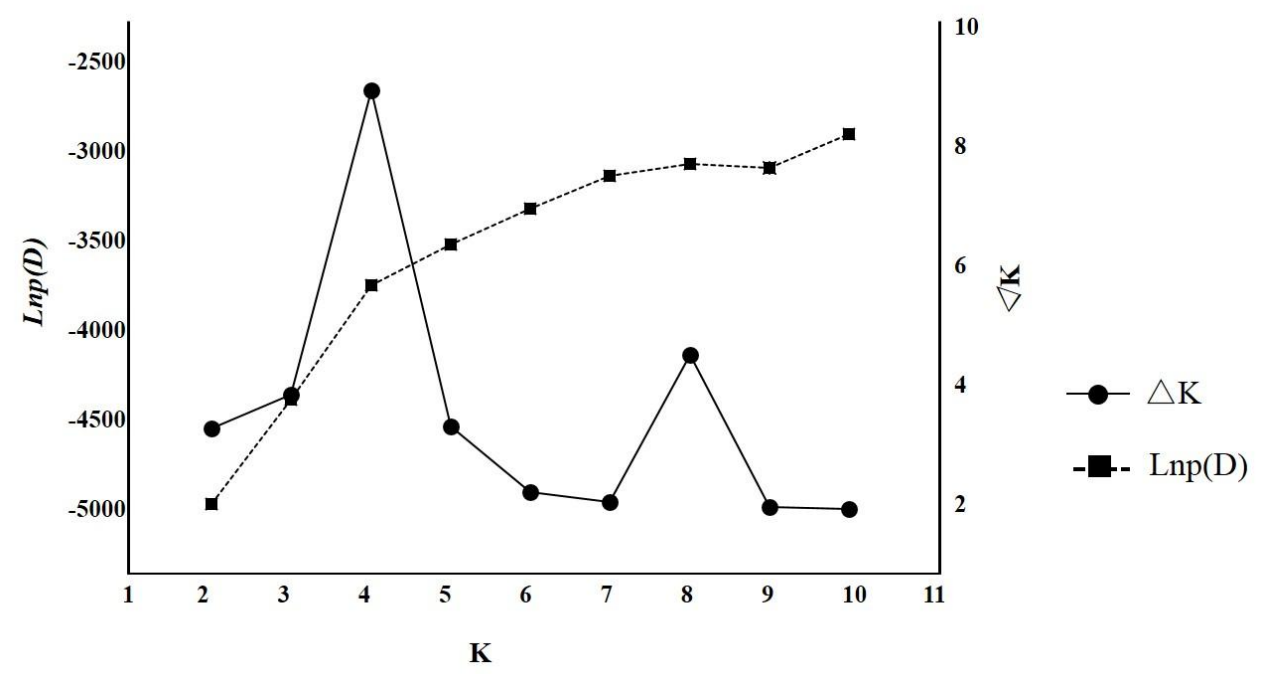

Figure 2. Plot of $\operatorname{Ln} P(D)$ and $\triangle K$ calculated for $K$ ranging from 1 to 10 
Based on the maximum membership probability, 98 inbred lines were assigned into 4 subpopulations (Reid, Lancaster, P, Domestic) (Fig.3). Reid included 35 inbred lines closed to B73 (Reid) genetic background. Lancaster had 26 inbred lines related to Mo17 (Lancaster) genetic background. P comprised 22 inbred lines, most derived from Pioneer hybrids (P). The other 15 inbred lines were closed to Dan 340 or Huangzao 4 background, which named as Domestic subgroup described by Xie et al. (2008).

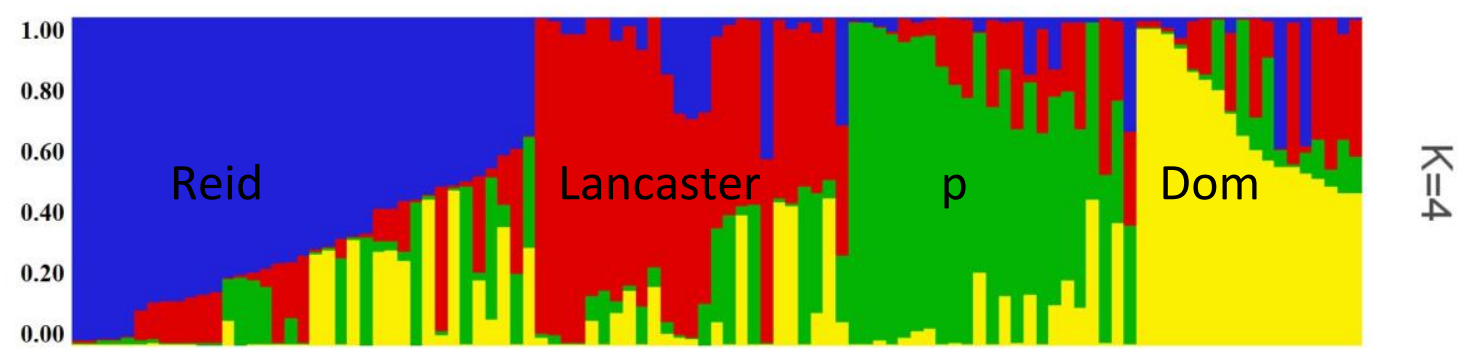

Figure 3. Population structure of the 98 inbred lines $(K=4)$. Note: The horizontal coordinate represents the 98 inbred lines, and the vertical coordinate of each subgroup indicates the membership coefficients for each individual. Blue zone: Reid; Red zone: Lancaster; Green zone: P; Yellow zone: Domestic

The similarity coefficient among 98 maize inbred lines ranged from 0.2692 to 0.9825 and the average was 0.6564 . When the similarity coefficient was 0.57 , UPGMA cluster analysis also clearly grouped 98 inbred lines into four subpopulations (Fig.4). Reid, Lancaster P and Domestic subpopulation comprised 36, 32, 13 and 17 inbred lines, respectively. The assignments of 77 inbred lines (78.6\% of the total) by UPGMA clustering were consistent with their assignments using STRUCTURE.

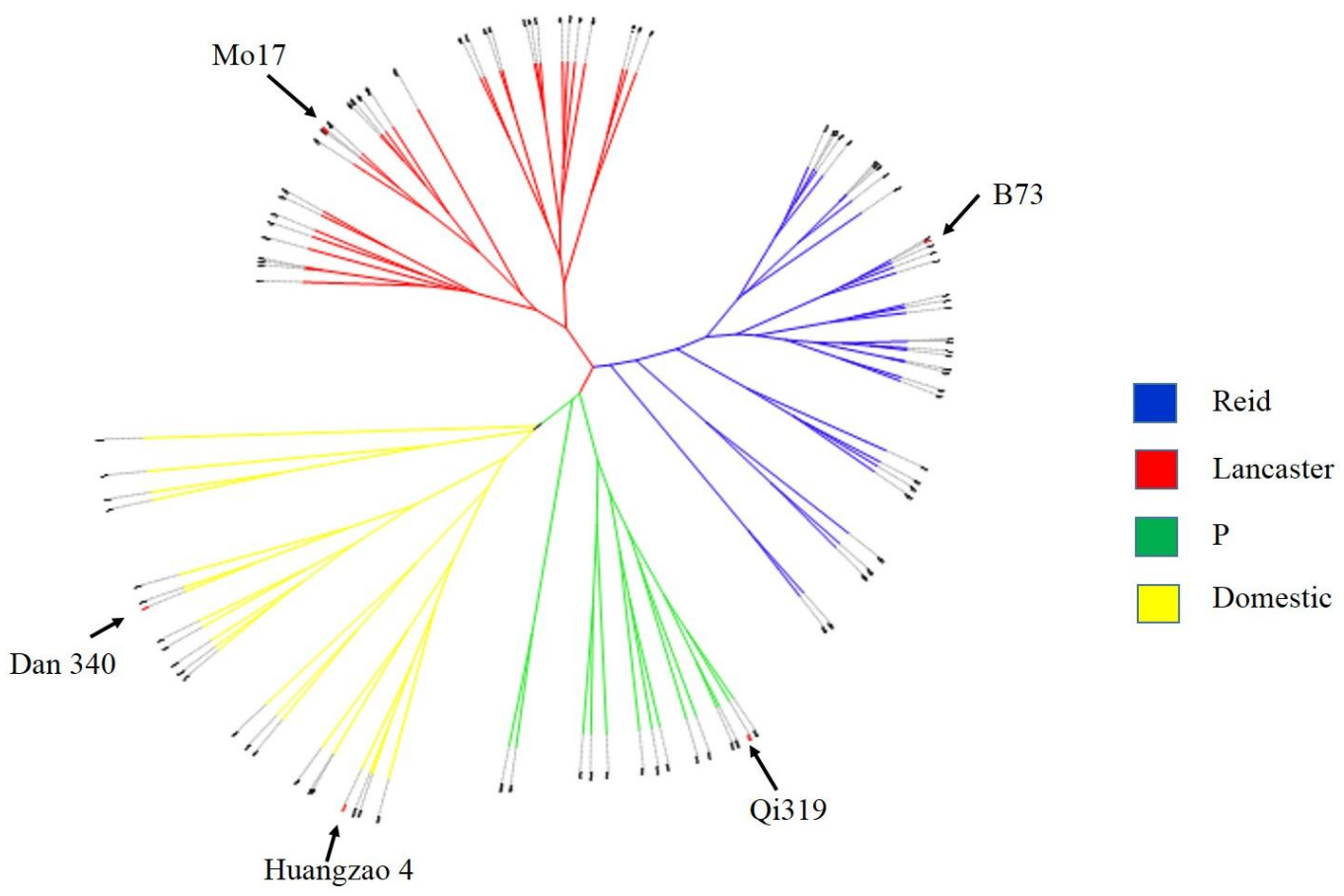

Figure 4. Dendrogram of 98 maize inbred lines based on UPGMA cluster analysis 
The principal coordinate analysis (PCoA) based on 145 SSR markers also separated the 98 maize inbred lines into four major groups (Fig.5). As inferred by STRUCTURE analysis, the inbred lines in Reid were mainly distributed in the lower left of the plot resulting, Lancaster distributed in the upper right, $\mathrm{P}$ in the upper left, and Domestic in the upper right. Most individuals within Reid and Lancaster subpopulations were grouped more closely. The inbred lines in P and Domestic were widely scattered, indicating that higher genetic diversity resided in $\mathrm{P}$ and Domestic subpopulations.

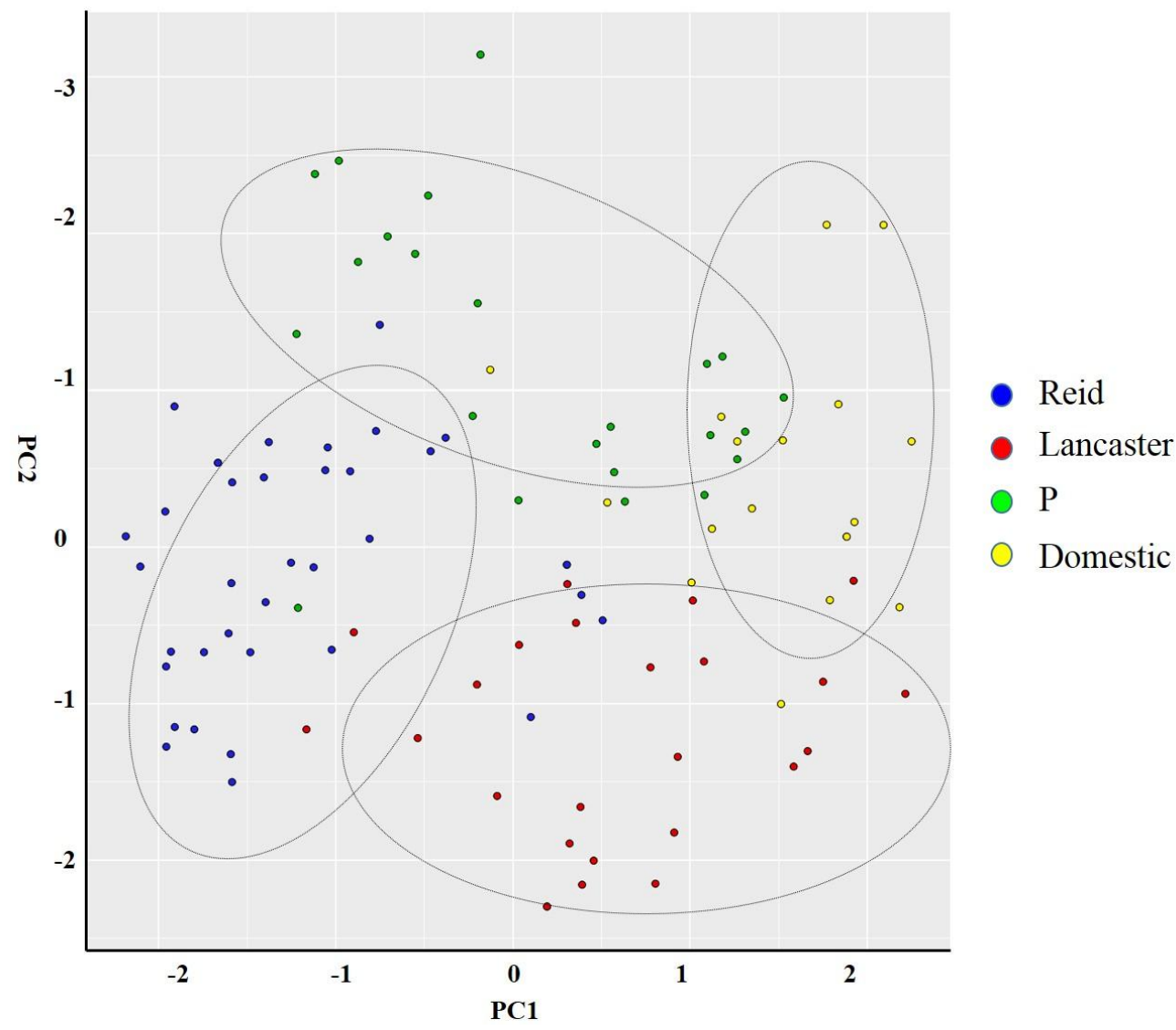

Figure 5. Principal coordinate analysis of 98 maize inbred lines based on 145 SSR markers. Reid (blue), Lancaster (red), P (green) and Domestic (yellow) are the four subgroups identified by STRUCTURE assigned with the maximum membership probability

\section{Discussion and Conclusion}

SSR markers, due to their abundance, co-dominance, and locus specificity, have been extensively used to assess genetic diversity in maize genotypes (Šarčević et al., 2008; Inghelandt et al., 2010).

In the present study, all the 98 tested maize inbred lines were derived from foreign hybrid-selected lines. We used 145 SSR markers to screen the population and a total of 450 alleles, with an average of 3.103 , were detected. The average polymorphic information content (PIC) value was 0.5067, which was lower than that in Chinese important inbred lines with PIC over 0.6 (Wang et al., 2008; Xie et al., 2008). The number of alleles found in this study is also in agreement with other studies (Wang et al., 2008; Park et al., 2015). Wang et al. (2008) reported a total of 1,365 alleles with an 
average of 9.4 alleles per locus by screening 95 inbred lines using SSR markers. Park et al. (2015) genotyped 174 maize inbred lines by 150 SSR markers and detected a total of 1082 alleles with an average of 7.21 alleles per locus. In our study, alleles were obtained at the whole genome level (Table 1). Chromosome 1 showed the highest allele number (59 alleles) and chromosome 8 the lowest (33 alleles). Therefore, we have thus determined that there is a higher level genetic diversity in the 98 foreign hybrid-selected lines, which has the potential to enhance the genetic diversity of Chinese maize breeding materials.

Population structure in the present study was also investigated using three complementary analysis methods STRUCTURE, UPGMA and PCoA based on SSR data. We selected maximum membership probability as the subgroup subdivision criterion, 98 maize inbred lines were assigned into four subpopulations which was in agreement with the assignments obtained by UPGMA and PCoA clustering. Nevertheless, for the 98 tested foreign hybrid-selected lines, the pedigree information was not in accordance with their clustering. In our data, the Domestic subgroup closed to Dan 340 or Huangzao 4 background. This finding can be partially explained by complex genetic background in foreign hybrids. Therefore, it is of significant importance to understand population structure and relationships among inbred lines is for maize improvement.

The allele frequencies, gene diversity and population structure obtained in the present study lead us to conclude that the 98 inbred lines derived from foreign hybridselected lines contain extensive genetic variation and are a valuable resource for Chinese maize breeding.

Acknowledgments. The research was partly supported by the projects of Shenyang international cooperation program (F15-200-6-02 and F16-221-6-00).

\section{REFERENCES}

[1] Abakemal, D., Hussein, S., Derera, J., Semagn, K. (2015): Genetic purity and patterns of relationships among tropical highland adapted quality protein and normal maize inbred lines using microsatellite markers. -- Euphytica 204: 49-61.

[2] Delphine, V. I., Albrecht, E. M., Claude, L., Benjamin, S. (2010): Population structure and genetic diversity in a commercial maize breeding program assessed with SSR and SNP markers. -- Theor Appl Genet 120: 1289-1299.

[3] Evanno, G., Regnaut, S., Goudet, J. (2005): Detecting the number of clusters of individuals using the software STRUCTURE: a simulation study. -- Mol. Ecol. 14: 26112620.

[4] Liu, K., Muse, S. V. (2005): PowerMarker: An integrated analysis environment for genetic marker analysis. -- Bioinformatics 21: 2128-2129.

[5] Park, J. Y., Ramekar, R. V., Sa, K. J., Lee, J. K. (2015): Genetic diversity, population structure, and association mapping of biomass traits in maize with simple sequence repeat markers. -- Genes Genom. 37: 725-735.

[6] Phumichai, C., Chunwongse, J., Jampatong, S., Grudloyma, P., Pulam, T., Doungchan, W., Wongkaew, A., Kongsiri, N. (2012): Detection and integration of gene mapping of downy mildew resistance in maize inbred lines though linkage and association. -Euphytica 187: 369-379.

[7] Pritchard, J. K., Stephens, M., Donnelly, P. (2000): Inference of population structure using multilocus genotype data. -- Genetics 155: 945-959. 
[8] Rohlf, F. J. (2009): NTSYSpc: numerical taxonomy and multi-variate analysis system. -Exeter Software, New York.

[9] Saghai-Maroof, M. A., Soliman, K. M., Jorgensen, R. A., Allard, R. W. (1984): Ribosomal DNA spacer length polymorphisms in barley: mendelian inheritance, chromosomal location, and population dynamics. -- Proc. Natl Acad. Sci. USA 81: 80148018.

[10] Šarčević, H., Pejić, I., Barić, M., Kozumplik, V. (2008): Originality of M3S maize population and changes in allele frequencies revealed by SSR markers after two cycles of selfed progeny recurrent selection. -- Euphytica 161: 97-105.

[11] Semagn, K., Magorokosho, C., Ogugo, V., Makumbi, D., Marilyn, Warburton, L. (2014): Genetic relationships and structure among open-pollinated maize varieties adapted to eastern and southern Africa using microsatellite markers. - Mol Breeding 34: 1423-1435.

[12] Tarter, J. A., Goodman, M. M., Holland, J. B. (2004): Recovery of exotic alleles in semiexotic maize inbreds derived from crosses between Latin American accessions and a temperate line. -- Theor Appl Genet 109: 609-617.

[13] Wang, R., Yu, Y., Zhao, J., Shi, Y., Song, Y., Wang, T., Li, Y. (2008): Population structure and linkage disequilibrium of a mini core set of maize inbred lines in China. -Theor Appl Genet. 117: 1141-1153.

[14] Wen, W., Guo, T., Victor, H., Tovar, C., Li, H., Yan, J., Taba, S. (2012): The strategy and potential utilization of temperate germplasm for tropical germplasm improvement: a case study of maize (Zea mays L.). -- Mol Breeding 29: 951-962.

[15] Wende, A., Shimelis, H., Derera, J., Mosisa, W., Danson, J., Laing, M. D. (2013): Genetic interrelationships among medium to late maturing tropical maize inbred lines using selected SSR markers. -- Euphytica191: 269-277.

[16] Xie, C., Warburton, M., Li, M., Li, X., Xiao, M., Hao, Z., Zhao, Q., Zhang, S. (2008): An analysis of population structure and linkage disequilibrium using multilocus data in 187 maize inbred lines. -- Mol Breeding. 21: 407-418.

[17] Yang, L., Wang, W., Yang, W., Wang, M. (2013): Marker-assisted selection for pyramiding the waxy and opaque-16 genes in maize using cross and backcross schemes. -- Mol Breeding 31: 767-775.

[18] Yong, H., Zhang, X., Zhang, D., Wang, J., Zhang, H., Li, M., Liu, W., Weng, J., Hao, Z., Ci, X., Ba, L., Li, X., Zhang, S. (2013): Breeding potential of U.S. maize germplasm for utilization in Chinese temperate conditions. -- Euphytica 192: 435-451.

[19] Živanović, T., Branković, G., Zorić, M., Momirović, G. S., Janković, S., Vasiljević, S., Pavlov, J. (2012): Effect of recombination in the maize breeding population with exotic germplasm on the yield stability. - Euphytica 185: 407-417. 


\section{APPENDIX}

Appendix 1. Germplasm sources and pedigrees of 103 maize acessions

\begin{tabular}{|c|c|c|}
\hline ID & Inbred line & Pedigree/source \\
\hline 1 & Huangzao4 & Landrace Tangsipingtou \\
\hline 2 & Mo17 & $\mathrm{C} 103 \times 187-2$ \\
\hline 3 & Dan340 & Baigulu $9 \times Z$ Z mays-tunicata \\
\hline 4 & Qi319 & selected from Pioneer hybrid '“78599' \\
\hline 5 & B73 & Iowa Stiff Stalk Synthetic C5 (BSSS) \\
\hline 6 & Y005 & selected from American hybrid "DK516" \\
\hline 7 & Y025 & selected from American hybrid "DK516" \\
\hline 8 & Y052 & selected from American hybrid "DK516" \\
\hline 9 & Y057 & selected from American hybrid "DK516" \\
\hline 10 & Y058 & selected from American hybrid "DK516" \\
\hline 11 & Y087 & selected from American hybrid "DK516" \\
\hline 12 & Y088 & selected from American hybrid "DK516" \\
\hline 13 & Y097 & unknown \\
\hline 14 & Y099 & unknown \\
\hline 15 & Y101 & unknown \\
\hline 16 & Y102 & unknown \\
\hline 17 & Y113 & selected from American hybrid "DK007" \\
\hline 18 & Y136 & selected from American hybrid "DK007" \\
\hline 19 & Y187 & selected from American hybrid "DK007" \\
\hline 2 & Y195 & selected from American hybrid "DK007" \\
\hline 21 & Y199 & selected from American hybrid "DK007" \\
\hline 22 & Y210 & selected from American hybrid "DK007" \\
\hline 23 & Y219 & selected from American hybrid "DK008" \\
\hline 24 & Y220 & selected from American hybrid "DK008" \\
\hline 25 & $\mathrm{Y} 255$ & selected from American hybrid "DK008" \\
\hline 26 & Y263 & selected from American hybrid "DK008" \\
\hline 27 & Y275 & selected from American hybrid "DK008" \\
\hline 28 & Y282 & selected from American hybrid "DK008" \\
\hline 29 & Y295 & selected from American hybrid "DK008" \\
\hline 30 & Y302 & selected from an American hybrid \\
\hline 31 & Y316 & selected from an American hybrid \\
\hline 32 & Y323 & selected from an American hybrid \\
\hline 33 & Y354 & selected from American hybrid " 3425 ” \\
\hline 34 & Y361 & selected from American hybrid " 3425 ” \\
\hline 35 & Y362 & selected from American hybrid " 3425 ” \\
\hline 36 & Y386 & selected from American hybrid " 3425 ” \\
\hline 37 & Y393 & selected from Pioneer hybrid "XY335" \\
\hline 38 & Y403 & selected from Pioneer hybrid "XY335” \\
\hline 39 & Y415 & selected from Pioneer hybrid "XY335" \\
\hline 40 & Y416 & selected from Pioneer hybrid "XY335” \\
\hline 41 & Y420 & selected from Pioneer hybrid "XY335" \\
\hline 42 & Y421 & selected from Pioneer hybrid "XY335" \\
\hline 43 & Y426 & selected from Pioneer hybrid "XY335" \\
\hline 44 & Y431 & selected from Pioneer hybrid "XY335" \\
\hline 45 & Y437 & selected from Pioneer hybrid "XY335" \\
\hline 46 & Y443 & selected from Pioneer hybrid "XY335" \\
\hline 47 & Y464 & selected from Pioneer hybrid "XY335" \\
\hline
\end{tabular}




\begin{tabular}{|c|c|c|}
\hline 48 & Y467 & selected from Pioneer hybrid "XY335” \\
\hline 49 & Y505 & selected from Pioneer hybrid "PR3394" \\
\hline 50 & Y509 & selected from Pioneer hybrid "PR3394" \\
\hline 51 & Y525 & selected from Pioneer hybrid "PR3394" \\
\hline 52 & Y531 & selected from Pioneer hybrid "PR3394" \\
\hline 53 & Y532 & selected from Pioneer hybrid "PR3394" \\
\hline 54 & Y569 & unknown \\
\hline 55 & Y570 & unknown \\
\hline 56 & Y594 & selected from American hybrid“"3382” \\
\hline 57 & Y625 & selected from American hybrid “'3382” \\
\hline 58 & Y645 & selected from American hybrid " 3382 " \\
\hline 59 & Y647 & selected from American hybrid " 3382 " \\
\hline 60 & Y648 & selected from American hybrid " 3382 " \\
\hline 61 & Y649 & selected from American hybrid " 3382 " \\
\hline 62 & Y683 & selected from Pioneer hybrid "XY222" \\
\hline 63 & Y700 & selected from Pioneer hybrid "XY222" \\
\hline 64 & Y721 & selected from Pioneer hybrid "XY222" \\
\hline 65 & Y728 & selected from Pioneer hybrid "XY222" \\
\hline 66 & Y733 & unknown \\
\hline 67 & Y751 & unknown \\
\hline 68 & Y753 & unknown \\
\hline 69 & Y760 & selected from Pioneer hybrid ' 78599 ' \\
\hline 70 & Y764 & selected from Pioneer hybrid " 78599 ' \\
\hline 71 & Y769 & selected from Pioneer hybrid ' 78599 ' \\
\hline 72 & Y777 & selected from Pioneer hybrid " 78599 ' \\
\hline 73 & Y793 & selected from Pioneer hybrid ' 78599 ' \\
\hline 74 & Y815 & selected from Pioneer hybrid ' 78599 ' \\
\hline 75 & Y829 & selected from Pioneer hybrid "XY508" \\
\hline 76 & Y837 & selected from Pioneer hybrid "XY508" \\
\hline 77 & Y839 & selected from Pioneer hybrid "XY508" \\
\hline 78 & Y851 & selected from Pioneer hybrid "XY508" \\
\hline 79 & Y882 & selected from Pioneer hybrid "XY508" \\
\hline 80 & Y886 & selected from Pioneer hybrid "XY508" \\
\hline 81 & Y889 & selected from Pioneer hybrid "XY508" \\
\hline 82 & Y895 & selected from Pioneer hybrid "XY508" \\
\hline 83 & Y897 & selected from Pioneer hybrid "XY508" \\
\hline 84 & Y918 & selected from Pioneer hybrid "33G35" \\
\hline 85 & Y919 & selected from Pioneer hybrid "33G35" \\
\hline 86 & Y920 & selected from Pioneer hybrid "33G35" \\
\hline 87 & Y963 & selected from Pioneer hybrid "33G35" \\
\hline 88 & Y966 & selected from Pioneer hybrid "33G35" \\
\hline 89 & Y1026 & selected from Pioneer hybrid "33G35" \\
\hline 90 & Y1043 & selected from Pioneer hybrid " 32 T24" \\
\hline 91 & Y1045 & selected from Pioneer hybrid " 32 T24" \\
\hline 92 & Y1052 & unknown \\
\hline 93 & Y1069 & unknown \\
\hline 94 & Y1071 & unknown \\
\hline 95 & Y1081 & unknown \\
\hline 96 & Y1084 & selected from Pioneer hybrid " 33 F20" \\
\hline 97 & Y1092 & selected from Pioneer hybrid " 33 F20" \\
\hline 98 & Y1094 & selected from Pioneer hybrid "32D22" \\
\hline 99 & Y1099 & selected from Pioneer hybrid "32D22" \\
\hline 100 & Y1115 & selected from Pioneer hybrid "32D22" \\
\hline
\end{tabular}




\begin{tabular}{l|l|l}
\hline 101 & Y1124 & selected from Pioneer hybrid "32D22" \\
102 & Y1161 & selected from Pioneer hybrid "32D22" \\
103 & Y1183 & selected from Pioneer hybrid "32D22" \\
\hline
\end{tabular}

Appendix 2. Chromosome locations for 145 SSRs, and their allele numbers and PIC value detected in 98 inbred lines of maize

\begin{tabular}{|c|c|c|c|c|c|c|c|}
\hline $\begin{array}{l}\text { Primer } \\
\text { Name }\end{array}$ & Bin & $\begin{array}{l}\text { No. of } \\
\text { alleles }\end{array}$ & PIC & $\begin{array}{l}\text { Primer } \\
\text { Name }\end{array}$ & Bin & $\begin{array}{l}\text { No. of } \\
\text { alleles }\end{array}$ & PIC \\
\hline umc985 & 1.06 & 6 & 0.7085 & umc 2400 & 5.04 & 3 & 0.4399 \\
\hline umc1166 & 1.02 & 2 & 0.4301 & bnlg389 & 5.09 & 4 & 0.5027 \\
\hline umc1243 & 1.04 & 2 & 0.3888 & bnlg1046 & 5.03 & 3 & 0.4637 \\
\hline umc1292 & 1.01 & 3 & 0.4136 & bnlg1118 & 5.07 & 3 & 0.4752 \\
\hline umc1358 & 1.07 & 2 & 0.3291 & bnlg1237 & 5.05 & 5 & 0.6998 \\
\hline umc1383 & 1.08 & 2 & 0.3082 & bnlg2323 & 5.04 & 2 & 0.3004 \\
\hline umc1568 & 1.02 & 3 & 0.5166 & umc1250 & 6.05 & 4 & 0.4149 \\
\hline umc1590 & 1.06 & 4 & 0.5438 & umc1296 & 6.06 & 2 & 0.3681 \\
\hline umc1706 & 1.07 & 3 & 0.5156 & umc1413 & 6.05 & 4 & 0.6408 \\
\hline umc1744 & 1.11 & 2 & 0.3860 & umc1474 & 6.05 & 2 & 0.2240 \\
\hline umc1972 & 1.06 & 2 & 0.3636 & umc1490 & 6.07 & 2 & 0.3697 \\
\hline umc2012 & 1.01 & 4 & 0.6776 & umc1653 & 6.07 & 5 & 0.7820 \\
\hline umc2025 & 1.05 & 3 & 0.4921 & umc1656 & 6.02 & 3 & 0.4869 \\
\hline umc 2240 & 1.08 & 2 & 0.3318 & umc1887 & 6.03 & 2 & 0.4445 \\
\hline bnlg439 & 1.03 & 4 & 0.6564 & umc1918 & 6.04 & 3 & 0.5316 \\
\hline bnlg1014 & 1.01 & 3 & 0.4587 & umc2006 & 6.04 & 3 & 0.4398 \\
\hline bnlg 1025 & 1.07 & 4 & 0.5626 & umc2059 & 6.08 & 2 & 0.4585 \\
\hline bnlg1866 & 1.03 & 6 & 0.8137 & umc2165 & 6.07 & 4 & 0.5976 \\
\hline bnlg2238 & 1.04 & 2 & 0.4073 & umc2312 & 6.01 & 3 & 0.3758 \\
\hline umc1026 & 2.04 & 2 & 0.4426 & bnlg107 & 6.01 & 4 & 0.5818 \\
\hline umc1265 & 2.02 & 2 & 0.3364 & bnlg161 & 6.00 & 5 & 0.7235 \\
\hline umc1419 & 2.00 & 3 & 0.4313 & bnlg1538 & 6.01 & 3 & 0.4943 \\
\hline umc1755 & 2.06 & 3 & 0.5509 & umc1066 & 7.01 & 2 & 0.4273 \\
\hline umc1845 & 2.03 & 3 & 0.5324 & umc1159 & 7.01 & 4 & 0.6501 \\
\hline umc1875 & 2.06 & 4 & 0.6769 & umc1213 & 7.02 & 2 & 0.4040 \\
\hline umc2094 & 2.01 & 5 & 0.7983 & umc1407 & 7.05 & 3 & 0.6391 \\
\hline umc2129 & 2.07 & 4 & 0.6692 & umc1593 & 7.03 & 7 & 0.6825 \\
\hline umc2214 & 2.10 & 2 & 0.3260 & umc1642 & 7.00 & 3 & 0.6911 \\
\hline umc 2372 & 2.06 & 4 & 0.5199 & umc1718 & 7.03 & 2 & 0.2511 \\
\hline bnlg1017 & 2.02 & 3 & 0.4619 & umc1782 & 7.04 & 5 & 0.7452 \\
\hline bnlg 1258 & 2.08 & 5 & 0.7293 & umc1799 & 7.06 & 4 & 0.6917 \\
\hline bnlg 1520 & 2.09 & 3 & 0.4612 & umc1944 & 7.04 & 2 & 0.4672 \\
\hline umc1501 & 3.05 & 2 & 0.3027 & umc1983 & 7.02 & 2 & 0.4719 \\
\hline umc1539 & 3.05 & 4 & 0.6191 & umc2057 & 7.07 & 3 & 0.4930 \\
\hline umc1886 & 3.02 & 3 & 0.5347 & bnlg1666 & 7.04 & 7 & 0.8316 \\
\hline
\end{tabular}




\begin{tabular}{|c|c|c|c|c|c|c|c|}
\hline umc2049 & 3.01 & 2 & 0.3834 & bnlg2132 & 7.00 & 2 & 0.3683 \\
\hline umc2118 & 3.00 & 2 & 0.4951 & umc1130 & 8.05 & 2 & 0.4149 \\
\hline umc 2263 & 3.04 & 3 & 0.4672 & umc1139 & 8.01 & 2 & 0.3220 \\
\hline umc2266 & 3.06 & 2 & 0.4086 & umc1161 & 8.06 & 2 & 0.4546 \\
\hline umc 2273 & 3.07 & 3 & 0.5603 & umc1384 & 8.07 & 3 & 0.5105 \\
\hline umc 2369 & 3.03 & 2 & 0.4614 & umc1530 & 8.03 & 3 & 0.4855 \\
\hline bnlg197 & 3.06 & 5 & 0.6653 & umc1638 & 8.09 & 2 & 0.5568 \\
\hline bnlg 1350 & 3.08 & 4 & 0.6432 & umc1663 & 8.09 & 3 & 0.4956 \\
\hline bnlg1496 & 3.09 & 5 & 0.6669 & umc1728 & 8.06 & 2 & 0.2907 \\
\hline bnlg 1754 & 3.09 & 5 & 0.7816 & umc1846 & 8.05 & 4 & 0.4745 \\
\hline bnlg2241 & 3.06 & 5 & 0.7553 & umc1984 & 8.03 & 2 & 0.4445 \\
\hline umc1017 & 4.01 & 3 & 0.5118 & bnlg666 & 8.05 & 4 & 0.5909 \\
\hline umc 1132 & 4.08 & 3 & 0.6059 & bnlg1863 & 8.03 & 2 & 0.4831 \\
\hline umc1294 & 4.02 & 2 & 0.3234 & bnlg2046 & 8.04 & 2 & 0.3315 \\
\hline umc1716 & 4.11 & 3 & 0.5778 & umc1107 & 9.04 & 3 & 0.5137 \\
\hline umc 1738 & 4.10 & 2 & 0.4949 & umc1271 & 9.03 & 2 & 0.2130 \\
\hline umc1821 & 4.04 & 2 & 0.4281 & umc1277 & 9.07 & 2 & 0.2976 \\
\hline umc1847 & 4.07 & 2 & 0.2759 & umc1366 & 9.06 & 3 & 0.3711 \\
\hline umc1943 & 4.02 & 3 & 0.4133 & umc1492 & 9.04 & 2 & 0.4905 \\
\hline umc2027 & 4.06 & 3 & 0.5921 & umc1505 & 9.08 & 2 & 0.3351 \\
\hline umc2039 & 4.03 & 2 & 0.4409 & umc1570 & 9.04 & 2 & 0.3240 \\
\hline umc2046 & 4.09 & 3 & 0.5153 & umc2128 & 9.03 & 3 & 0.5502 \\
\hline umc 2188 & 4.08 & 2 & 0.5254 & umc2337 & 9.03 & 3 & 0.4308 \\
\hline bnlg 1265 & 4.05 & 3 & 0.4761 & umc2343 & 9.05 & 5 & 0.7501 \\
\hline bnlg1444 & 4.08 & 5 & 0.7055 & bnlg1191 & 9.07 & 4 & 0.4862 \\
\hline bnlg1621 & 4.06 & 4 & 0.5840 & bnlg1401 & 9.02 & 4 & 0.6455 \\
\hline bnlg1890 & 4.11 & 4 & 0.6944 & umc1196 & 10.07 & 3 & 0.5811 \\
\hline bnlg 2162 & 4.08 & 5 & 0.6742 & umc1319 & 10.01 & 3 & 0.5145 \\
\hline umc1019 & 5.06 & 6 & 0.7887 & umc1380 & 10.00 & 2 & 0.4580 \\
\hline umc1072 & 5.07 & 3 & 0.6536 & umc1477 & 10.05 & 4 & 0.5459 \\
\hline umc1389 & 5.03 & 2 & 0.3367 & umc1506 & 10.05 & 3 & 0.5425 \\
\hline umc 1478 & 5.01 & 2 & 0.4445 & umc1873 & 10.04 & 2 & 0.4724 \\
\hline umc1624 & 5.04 & 2 & 0.3501 & umc1877 & 10.07 & 3 & 0.2772 \\
\hline umc1692 & 5.03 & 2 & 0.4182 & umc2069 & 10.02 & 4 & 0.5141 \\
\hline umc2036 & 5.01 & 3 & 0.5573 & umc2350 & 10.04 & 2 & 0.4190 \\
\hline umc 2136 & 5.08 & 3 & 0.6215 & bnlg987 & 10.03 & 4 & 0.5482 \\
\hline umc2216 & 5.06 & 4 & 0.5698 & bnlg2190 & 10.06 & 4 & 0.7093 \\
\hline umc 2386 & 5.05 & 2 & 0.4352 & & & & \\
\hline
\end{tabular}

Note: Bin numbers were determined from maizeGDB 